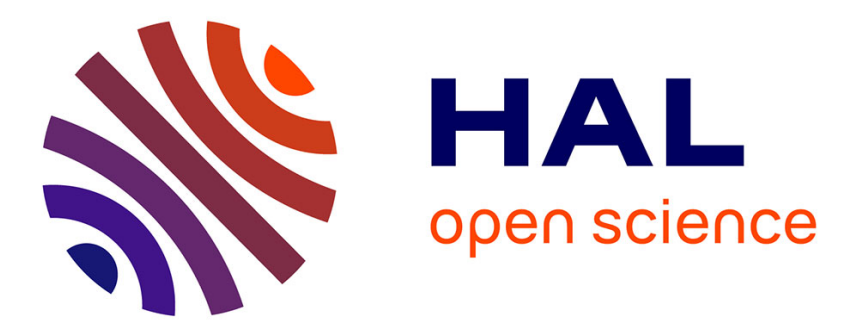

\title{
Détermination des longueurs d'onde des radiations très réfrangibles du magnésium, du cadmium, du zinc et de l'aluminium
}

\author{
A. Cornu
}

\section{- To cite this version:}

A. Cornu. Détermination des longueurs d'onde des radiations très réfrangibles du magnésium, du cadmium, du zinc et de l'aluminium. J. Phys. Theor. Appl., 1881, 10 (1), pp.425-431. 10.1051/jphystap:0188100100042501 . jpa-00237838

\section{HAL Id: jpa-00237838 https://hal.science/jpa-00237838}

Submitted on 1 Jan 1881

HAL is a multi-disciplinary open access archive for the deposit and dissemination of scientific research documents, whether they are published or not. The documents may come from teaching and research institutions in France or abroad, or from public or private research centers.
L'archive ouverte pluridisciplinaire HAL, est destinée au dépôt et à la diffusion de documents scientifiques de niveau recherche, publiés ou non, émanant des établissements d'enseignement et de recherche français ou étrangers, des laboratoires publics ou privés. 
A. CORNU. - LONGUEURS D'ONDE DES RADIATIONS, ETC. 425

\section{DÉTERMINATION DES LONGUEURS D'ONDE DES RADIATIONS TRĖS RÉFRAN- GIBLES DU MAGNÉSIOM, DU CADMIUM, DU ZING ET DE L'ALUMINIOM;}

P.IR M. A. CORNU.

La détermination des longueurs d'onde par la méthode des réseaux est toujours une opération délicate lorsqu'on désire obtenir une certaine précision : la difficulté augmente beaucoup lorsqu'on se sert d'un goniomètre dont les objectifs ne sont pas achromatiques et que les sources de lumière dont on cherche la longueur d'onde ne donnent, après diffraction, que peu d'intensité. 
C'est à l'occasion de la détermination des longueurs d'onde du spectre solaire pour la construction de la Carte du spectre normal ultra-violet (') que jai construit des objectifs achromatiques formés d'une lentille convergente de quartz et d'une lentille divergente de spath d'Islande.

La description de ce système de lentilles a été donnée dans le Bulletin de la Société de Physique () et dans le présent Journal, เ. VIII, p. ı 85 . Ces objectifs permettent d'obtenir sur un même cliché d'enıiron $\mathrm{o}^{\mathrm{mm}}$, o4o de longueur la totalité du spectre solaire photographique, depuis la raie $G$ jusqu'à la raie $U$, avec une netteté très satisfaisante dans toute l'étendue du champ.

Mais les dispersions des deux substances n'ont pas entre elles les relations convenables pour établir un achromatisme très parfait; la variation de foyer devient notable pour les raies de réfrangibilité ćlevée; en outre, le spath d'Islande présente un pouvoir absorbant assez énergique pour les dernières raies observables, de sorte que ces objectifs ne conviennent plus pour l'étude des radiations les plus réfrangibles.

Jai découvert une substance au moins aussi transparente pour les rayons ultra-violets que le quartz, et qui possède une loi de dispersion si bien en harmonie avec celle du quartz qu'elle permet d'obtenir un système de lentilles dont l'achromatisme est presque parfait; cette substance est le spath-fluor incolore de Suisse.

Pour donner une idée de la perfection de l'achromatisme, il me suffira de dire que j'obtiens couramment sur le même cliché, avec une nettelé très satisfaisante, le spectre de toutes les raies photographiques des métaux, depuis les trois raies bleues du zinc, jusqu'aux raies $\mathrm{n}^{\circ} 32$ de l'aluminium.

Grâcè à ces objectifs, la détermination de la longueur d'onde des raies très réfrangibles devient abordable; roici le résumé de cette étude :

Mode d'observation. - Un excellent goniomètre de Brunner donnant les $3^{\prime \prime}$ porte une lunette et un collimateur d'environ $\mathrm{o}^{\mathrm{m}}, 43$ de longueur focale, munis d'objectifs quartz-spath-fluor, et sur la

(') Voir Comptes rendus des séances de l'Academie des Sciences, t. LXXXVI, p. 103 .

(') - février 1879 . 
LONGUEURS D'ONDE DES RADIATIONS TRÈS RÉFRANGIBLES. 42plate-forme centrale un beau réseau sur verre que je dois à l'obligeance de M. Rutherfurd, de New-Iork.

Ce réseau, qui était argenté lorsque je l'ai reçu, a étć dépouillé de la couche métallique de façon à laisser à nu la face striée; la face opposée avait été noircie pour éviter les réflexions étrangères; il fonctionnait par réflexion sous un angle d'incidence toujours voisin de $27^{\circ} 3 \mathrm{o}^{\prime}$, incidence que j’aj reconnue convenable pour observer au moins deux ou trois ordres de spectre de part et d'autre de l'image réfléchie. La partie striée du réseau, qui a la forme d'un carré d'environ $\mathrm{o}^{\mathrm{m}}, \mathrm{o}_{2} \mathrm{\jmath}$ de côté, envoie par diffraction une telle quantité de lumière que l'on observe sans peine, malgré l'absence d'argenture, les raies brillantes des étincelles d'induction, non seulement dans le spectre visible, mais encore dans le spectre ultraviolet, à l'aide de l'oculaire fluorescent de M. Soret; dans ce dernier cas la visibilité est faible, mais elle serait suffisante avec certains métaux pour effectuer des mesures. J'ai toutefois préféré l'emploi de la Photographie, qui fournit, sans aucune fatigue pour la vue de l'observateur, des clichés qu'on étudie ensuite à loisir.

La méthode d'observation est celle que j'ai décrite précédemment ( ${ }^{1}$ ) et qui consiste à conclure la déviation d'une raie de sa distance à deux repères obtenus avec l’image direcle (ici, réfléchie) de la fente; les deux repères donnent d'une part la valeur angulaire du cliché et de l'autre, par leur position moyenne, l'origine des déviations. Dans le cas des spectres de diffraction obtenus par réflexion d'un faisceau parallèle sous l’incidence $i$, la déviation d'ordre $n, \Delta_{n}$, comptée à partir du rayon réfléchi sur la surface plane du réseau, est liée à la longueur d'onde $\lambda$ et à la constante $a$ du réseau ( $\left.{ }^{2}\right)$ par la formule

$$
a\left[\sin i-\sin \left(i-\Delta_{n}\right)\right]=n \lambda,
$$

ou encore

$$
2 a \sin \frac{\Delta_{n}}{2} \cos \left(i-\frac{\Delta_{n}}{2}\right)=n \lambda .
$$

(') Annales de l'École Normale, $2^{\circ}$ série, t. III, p. $42 \mathrm{I}$.

(2) La constante du réseau a été déterminée par l'observation de la raie la plus réfrangible des raies $D$ avec la lumière de la soude $(\lambda=588,89)$ dans le premier spectre de droite et les deux premiers spectres de gauche; $j$ 'ai trouve $a=2935,8$ ou $\mathrm{o}^{\mathrm{mm}}, 0029358$. 
J'ai opéré avec les étincelles d'induction jaillissant entre deux électrodes métalliques en employant successivement le magnésium, le cadmium, le zinc et l'aluminium (contenant des traces de zinc); les Tableaux suivants donnent les résultats bruts selon l'ordre du spectre observé : la dernière colonne donne la moyenne composée, calculée d'après la méthode des moindres carrés, qui s'applique ici d'une manière extrèmement simple.

IIG YÉSIU II.

\begin{tabular}{|c|c|c|c|c|c|c|}
\hline \multirow{2}{*}{ DÉSIGYATION DE LI RAIE. } & \multirow{2}{*}{$\underset{1: e}{\text { DROITE }}$} & \multicolumn{4}{|c|}{ G.ALCHE } & \multirow{2}{*}{$\begin{array}{l}\text { Loxguevr } \\
\text { d'onde } \\
\text { adoptee. }\end{array}$} \\
\hline & & 110 & $2^{e}$ & $3 e$ & $4^{\mathrm{e}}$ & \\
\hline $\begin{array}{l}\text { La moins réfrangible de la } \\
\text { raie triple........ } \\
\text { Raie double }\left\{\begin{array}{l}\text { forte. } \ldots \ldots \\
\text { faible } \ldots \ldots\end{array}\right. \\
\text { Raie isolè } \ldots \ldots \ldots \ldots \ldots\end{array}$ & $\begin{array}{c}309,37 \\
29^{3}, 24 \\
292,50 \\
284,9 \mathrm{r} \\
\prime \prime \\
\prime \prime \\
\text { milieu du } \\
{[279,37]} \\
\prime \prime \\
\prime \prime\end{array}$ & $\begin{array}{c}309,85 \\
293,67 \\
292,83 \\
285,15 \\
\prime \prime \\
\prime \prime \\
\text { groupe } \\
{\left[\begin{array}{c}379,62 \\
\prime \prime \\
\prime \prime\end{array}\right]}\end{array}$ & $\begin{array}{l}\text { " } \\
\text { " } \\
\text { " } \\
\text { " } \\
\text { " }\end{array}$ & $\begin{array}{c}\prime \prime \\
29^{3}, 56 \\
29^{2}, 78 \\
\prime \prime \\
280,25 \\
279,79 \\
279,52 \\
279,07\end{array}$ & $\begin{array}{c}\prime \prime \\
29^{3,55} \\
29^{2}, 69 \\
\prime \prime \\
280,09 \\
279,71 \\
279,46 \\
278,98\end{array}$ & $\begin{array}{l}3 \circ 9,6 x \\
293,49 \\
292,67 \\
285, \text { o3 } \\
280,13 \\
279,7 \text { 1 }\end{array}$ \\
\hline
\end{tabular}

La raie quadruple du magnésium est la plus intense des raies qu'on puisse obtenir, toutes choses égales d'ailleurs, avec les métaux

Fig. 1 .

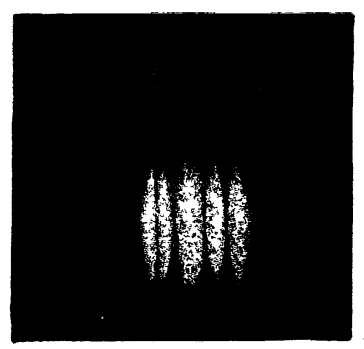

usuels. Elle présente une particularité tout à fait étrange : deux de ses composantes sont renversées, c'est-à-dire dédoublées, dès que la surface du condensateur qui donne l'énergie à l'étincelle d'in- 
duction est un peu étendue ; la figure ci-jointe est la reproduction d'une épreuve sur laquelle j'ai obtenu successivement sur le mème cliché l'image de la raie quadruple intense avec un fort condensateur. Dans le dernier cas les deux raies ne sont plus renversées et la plus faible des deux autres a presque disparu ; la comparaison ne laisse aucun doute sur l'explication de l'apparence étrange de cette raie quadruple, qui devient quintuple et sextuple suivant l'énergie de l'étincelle.

\section{CADMIUM.}

\begin{tabular}{|c|c|c|c|c|c|c|c|c|}
\hline \multirow{2}{*}{$\begin{array}{c}\text { Désigiva TION } \\
\text { de la raie. } \\
\text { (Notation de M Mascart }\end{array}$} & \multicolumn{3}{|c|}{ DROITE } & \multicolumn{4}{|c|}{ GAUCHE } & \multirow{2}{*}{$\begin{array}{l}\text { Loviceur } \\
\text { d'onde } \\
\text { adoptee. }\end{array}$} \\
\hline & $3 \mathrm{e}$ & $2 \mathrm{e}$ & 1er & 1er & $2 e$ & 3e. & 40 & \\
\hline $\mathbf{N}^{\mathrm{No}} 10 \ldots \ldots \ldots$ & $"$ & "I & $347,1 ?$ & " & $3,6,46$ & $1 "$ & $" 1$ & 346,68 \\
\hline $1 \pi \ldots \ldots$ & " & " & $2-5,49$ & 273,8 & " & $\left\{\begin{array}{l}274,59 \\
274,45\end{array}\right.$ & " & $\begin{array}{c}2,4,77 ? \\
\text { (probablement } \\
\text { un peu } \\
\text { trop forte.) }\end{array}$ \\
\hline $18 \ldots \ldots \ldots$ & 257,38 & $"$ & $"$ & $. .56,59$ & " & $.57,11$ & $" \prime$ & 257,23 \\
\hline $22 \ldots \ldots \ldots$ & 232,15 & " & " & " & " & 232,28 & 232,18 & 232,18 \\
\hline $23 \ldots \quad \ldots \ldots$ & $23 \mathrm{r}, 32$ & " & " & $23 \mathrm{I}, \mathrm{I}$ ? & 231,10 & 231,42 & $23 \mathrm{r}, 3-\mid$ & $.31,35$ \\
\hline Raie faible isolee. & $228,8 j$ & " & $"$ & " & $" \prime$ & $\prime \prime$ & $22 \leqslant, 8.5$ & 298,85 \\
\hline $\mathrm{N}^{\circ \mathrm{os}} 24 \ldots \ldots \ldots \ldots$ & 226,57 & " & $"$ & $\because 26,40$ & 226,31 & $226,58 \mid$ & $226,53 \mid$ & 226,55 \\
\hline $25 \ldots \ldots \ldots \ldots$ & 219,50 & "I & " & 219,10 & 219,30 & $2 \times 9,16$ & $\left\{\begin{array}{l}219,42 \\
2 \times 9,3 \gamma\end{array}\right\}$ & $219,4^{5}$ \\
\hline $26\left({ }^{1}\right) \ldots \ldots \ldots$ & 214,49 & "I & " & $\because 14,66$ & 214, 39 & 214,39 & $\begin{array}{l}219,3-1 \\
\mid 214,3-i \\
1\end{array}$ & ?.1多, \\
\hline
\end{tabular}

D'après une comparaison directe des raies $\mathrm{n}^{\mathrm{os}} 9,10,11$ et 12 du cadmium avec le spectre solaire, j'ai conclu par comparaison avec la Carte qui représente mes observations antérieures :

$$
\begin{aligned}
& \text { No } 9(\text { air }) \lambda=360,90 \text { (une rave faible à côté) } 36 \text { r. r- } \\
& \mathrm{N}^{\circ} 10 \quad 346,55 \ldots \ldots \ldots \ldots \ldots \ldots \ldots \ldots, 6,6 ; \\
& \mathrm{N}^{\circ} 11 \quad 3 \text { i } 15 \\
& \text { No } 12 \text { triple }\left\{\begin{array}{l}
325,8 \\
324,8 \\
324, ; \text { la plus forte. }
\end{array}\right.
\end{aligned}
$$

(') Pour obtenir des épreuves photographiques de la raie $n^{\circ} 26$ et des raies plus réfrangibles des autres métaux, au collodion humide, il est indispensable de laver la glace sensibilisée avec de l'eau distillée, car la solution d'azotate d'argent absorbe complètement les radiations très refrangibles, mème lorsqu'elle est en couche aussi mince. 
La concordance des résultats avec ceux de M. Mascart ( ${ }^{1}$ ) est satisfaisante, excepté pour les raies 12 et $2 \breve{3}$; j’ai examiné la question de très près et je crois mes déterminations exactes.

ZINC.

\begin{tabular}{|c|c|c|c|c|c|c|c|c|c|c|}
\hline \multirow{2}{*}{$\begin{array}{l}\text { DÉSIGX.ITIOX } \\
\text { de la raie. } \\
\text { (Notation } \\
\text { de II Soret }\end{array}$} & \multicolumn{3}{|c|}{ DROITE } & \multicolumn{6}{|c|}{ G.AccuE. } & \multirow{2}{*}{ 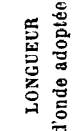 } \\
\hline & 3e. & 20 & 1er. & 1er. & 20. & $3 e$ & 40. & se. & $6 \theta$. & \\
\hline $\begin{array}{l}\text { Raie du } \mathrm{Zn} \text { près } \\
\text { du } \mathrm{n}^{\circ} \quad 26 \quad \text { du } \\
\text { cadmium.... }\end{array}$ & 213,88 & " & " & $3 \times 3,50$ & $2 \times 3,-5$ & $\because 3,81$ & " & " & " & 13,85 \\
\hline $\begin{array}{l}\text { Yo } 97 \text { (double } \\
\text { la plus forte. }\end{array}$ & $209 \cdot 92$ & " & " & 209,73 & $209,9^{6}$ & $309,99\}$ & $\left.\begin{array}{l}30,00 \\
209,91 \\
305,45\end{array}\right\}$ & 11 & $209,9^{\circ}$ & $.09,88$ \\
\hline Y.28 $\left\{\begin{array}{l}28_{1} \text { fuible. } \\
28_{2} \text { forte.. }\end{array}\right.$ & $\begin{array}{l}206,30 \\
206,06\end{array}$ & " & " & " & $\begin{array}{l}206,17 \\
206,20\end{array}$ & 206,1 任 & $\begin{array}{l}206,37 \\
206,21 \\
206,1\end{array} \mid$ & " & $\begin{array}{l}236,35 \\
206,12\end{array}$ & $\begin{array}{l}206,34 \\
206,10\end{array}$ \\
\hline No $29 \ldots \ldots \ldots$ & 202,34 & " & ' & 202,12 & 202,62 & 202,56 & $\mid \begin{array}{l}302,5-1 \\
302,9\end{array}$ & " & 102,45 & 02,43 \\
\hline
\end{tabular}

ALUMINIUM (avec traces de zinc).

\begin{tabular}{|c|c|c|c|c|c|c|c|}
\hline \multirow{2}{*}{$\begin{array}{l}\text { DÉsIGXatiox DE LA RAIC. } \\
\text { (Notation de } \mathrm{M} \text { soret) }\end{array}$} & \multicolumn{3}{|c|}{ DROITE } & \multicolumn{3}{|c|}{ GALCHE } & \multirow{2}{*}{$\begin{array}{c}\text { LONGLELR } \\
\text { d onde } \\
\text { adoptee. }\end{array}$} \\
\hline & $3 e$ & $2^{c}$. & 1 er. & 1 er. & $2 e$ & $3^{e}$. & \\
\hline$N^{o s} 29$ (zinc) $\ldots$ & $\begin{array}{l}202,02 \\
202,14\end{array}$ & " & "I & $\left\{\begin{array}{l}203,4^{\prime} t^{2} \\
203,41\end{array}\right.$ & $\mathfrak{i}^{\prime \prime}$ & " & $202,4^{2}$ \\
\hline 30. & $\begin{array}{l}198,46 \\
198,52\end{array}$ & " & 197,90 & $\begin{array}{r}199,9^{\mathrm{I}} \\
\mathbf{1} 99, \mathrm{~S}_{2}\end{array}$ & $l^{\prime \prime \prime}$ & $\begin{array}{l}199, \mathbf{I} \\
199, \mathbf{1} 4\end{array}$ & $\mathbf{r} 98,8 \mathrm{r}$ \\
\hline $31 \quad 31, \ldots \ldots$ & ${ }^{12}, 9^{6}$ & " & I 92,36 & $\left\{\begin{array}{r}194,58 \\
194,45\end{array}\right.$ & \}$"$ & $\begin{array}{l}19^{3}, 7^{2} \\
19^{3}, 7^{2}\end{array}$ & $19^{3,35}$ \\
\hline$\left(31_{2},\right.$. & 192,48 & " & " & 19', 198 & " & $19^{3,26}$ & 192,$8 ;$ \\
\hline $32, \ldots$ & $\begin{array}{l}180,54 \\
185,66\end{array}$ & $i^{\prime \prime}$ & $184,7 x$ & $\left\{\begin{array}{l}187,19 \\
1 \quad 187,07\end{array}\right.$ & \}$"$ & $\begin{array}{l}186,1^{\circ} \\
186,50\end{array}$ & 186,02 \\
\hline & $\begin{array}{l}184,75 \\
184,86\end{array}$ & " & 184, II & $\left\{\begin{array}{l}186,4^{2} \\
186,21\end{array}\right.$ & \{ & r 85,66 & 185,22 \\
\hline
\end{tabular}

La concordance paraît moins grande avec l'aluminium, entre

(') Annales de l'École Normale, $1^{\text {re }}$ série, t. IV.

(') Il y a une raie très faible entre $32_{1}$ et $32_{2}$. 
les valeurs déduites des spectres de droite et celles déduites du spectre de gauche; cela tient à une erreur systématique que jai sciemment mise en évidence dans le relevé des repères; cette erreur est causée par les imperfections du réseau, des réglages et surtout de l'achromatisme des objeclifs; en effet, l'impression de l'image des repères est produite par la somme des impressions de toutes les radiations; le foyer de toutes ces radiations n’étanl pas rigoureusement le même, les images des repères sont un peu estompées et légèrement dissymétriques; j’aurais dù pointer dans le milieu de l’image : j’ai cru plus exact de pointer sur l'un des bords, qui était assez net sur tous les clichés; aussi, pour faire disparaître l'effet de cette erreur systématique, qui accroissait la déviation de gauche et diminuait de la même quantité celle de droite, je me suis astreint à observer les mêrnes spectres à droite et à gauche, ce qui élimine la divergence : on peut s'en assurer en prenant les moyennes des résultats de même ordre. Comme vérification, on peut constater que l'erreur est sensiblement en raison inverse du numéro d'ordre des spectres.

Ce genre d'erreur est plus grand avec l'aluminium qu'avec les autres métaux, parce que les raies intenses sont réparties tout le long du spectre, y compris les plus réfrangibles de toutes, ce qui exagère les effets dus à l'imperfection de l'achromatisme des objectifs; l'effet était déjà sensible avec le cadmium, tandis qu'il l'est peu avec le zinc, parce que les raies $2 \mathbf{7}, 28,29$ sont extrêmement intenses. 